\title{
FORMALIZATION OF DESIGN PROCEDURES IN THE ENGINEER'S EDUCATIONAL STRATEGY
}

\author{
Nikolay M. Borgest \\ Samara state aerospace university \\ Samara, Russia \\ borgest@yandex.ru
}

\author{
Maksim D. Korovin \\ Samara state aerospace university \\ Samara, Russia \\ maks.korovin@gmail.com
}

\begin{abstract}
During the last seven years Samara State Aerospace University performs an educational strategy for engineers and masters programs in aircraft engineering. The goal of the strategy is to formalize procedures and operations within airplane designing activities, in particular, the preliminary airplane design. The ultimate goal of the strategy is to create an automated system that would be able to generate a preliminary design project for an airplane based on the given technical specification for the project.

The whole complex of standard design procedures, necessary to create an airplane, is carefully examined in order to achieve this goal. A domain thesaurus is being created as an integrative basis for the system. The system itself is based on a database of parameterized CAD models. It consists of a number of CAD models of different airplane parts, such as wing, fuselage, empennage, etc., for different types of airplanes and different design approaches. During the design process, the models are elaborated according to the chosen design scenario. The design scenarios are chosen based on their applicability for a particular task. If none of the existing scenarios is applicable, a new one is developed.
\end{abstract}

An interactive interface for the designing support system is being developed to demonstrate the decisions, made during the designing process and also to give an operator ability to interfere if necessary.

Students are involved in this program on the later years of their education, as it is a part of their graduation work. Each individual task is assigned to one of two students. All students, involved in the project, must cooperate with each other as their ultimate goal is to combine their effort into a single informational system. After completion of the individual goals, masters students prepare papers for specialized scientific conferences and, later, their masters thesis.

The paper summarizes the experience in airplane designer's education carried out with support of modern informational systems, programming languages, data and knowledge bases.

Keywords—design, automation, education, aircraft, CAD

\section{INTRODUCTION}

During the last seven years Samara State Aerospace University performs an educational strategy for engineers and masters programs in aircraft engineering. The goal of the strategy is to formalize procedures and operations within airplane designing activities, in particular, the preliminary airplane design stage.

The ultimate goal of the strategy is to create an automated system that would be able to generate a preliminary design project for an airplane based on the given technical specification for the project. In other words, to create a system, that would be able to perform the necessary tasks for creating a preliminary model of an aircraft, based on a traditional scheme.

\section{DOMAIN THESAURUS AS AN INTEGRATIVE BASIS FOR A DESIGNING SYSTEM}

The goal of the designed thesaurus is to serve as a semantic integrative basis in creation of the robot-designer. Implementation of a universal controlled dictionary allows to avoid or at least heavily diminish problems, caused by definitions ambiguity within the members of the group, involved in the project [1].

Domain thesaurus is a subclass of ontology and thus should be developed as one. The process of creation of thesaurus starts with the localization of a subject domain. In case of the robotdesigners' thesaurus, global subject domain is aircraft design, local subject domain - preliminary airplane design. Preliminary airplane design is a process of creation of a prototype project for a designed airplane. In order to create a thesaurus it is necessary to conduct a system analysis of the subject domain - to determine interconnections between elements of the studied system, to perform ontological analysis of the domain.

Basic terminology of the subject domain is taken from the FAR 25, student's books, terminological dictionaries, encyclopaedias, monographs and papers in the studied domain [2]. Ontology in the form of thesaurus serves to present information, necessary for understanding of the place of a particular term in the terminological environment.

The designed thesaurus should contain all essential definition of the subject domain. In case of the preliminary airplane design it should comprise different aspects of airplane design, study of its geometrical, mass and aerodynamic characteristics, flight equipment and flight trajectories.

During the design of thesaurus no size limitation were implemented. In its current state, as for early 2016, it contains 
1226 terms and definitions and 505 associative links. Students implement the thesaurus using special software, such as, for example, Protégé, or Fluent Editor. A fragment of the thesaurus implemented in the Fluent Editor is shown on figure 1.

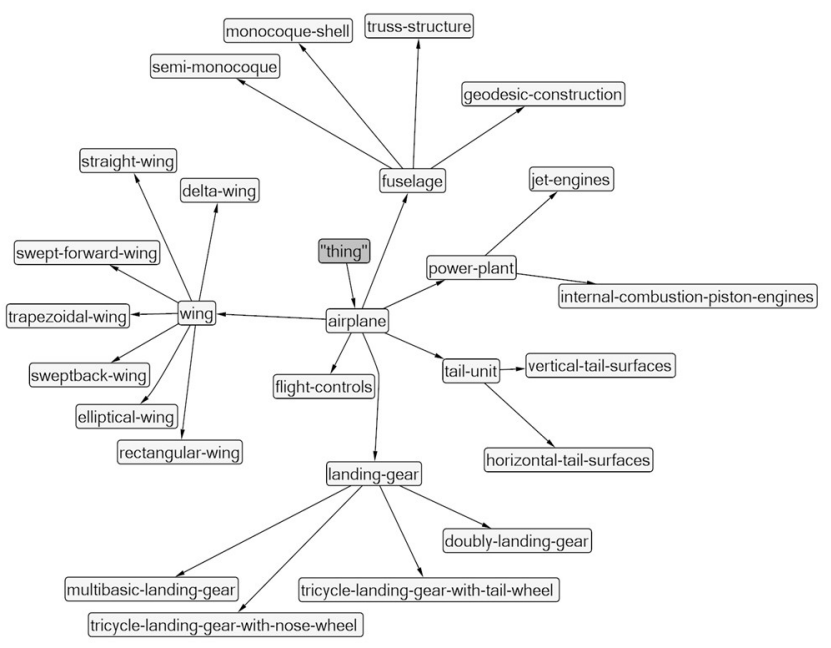

Fig. 1. Fragment of the "Preliminary airplane design" thesaurus

\section{ROBOT-DESIGNER'S OPERATION SCHEME}

The preliminary design stage involves the development of the general concept the designed object, the conceptual model of object elements, the formation of a feasibility study, the formation of job design, and preparation of design process. Description of the object formed on the preliminary design stage, includes constructive scheme, approximate estimates of weight and dimensions, power consumption and reliability [3].

In critical cases, manufacturing and testing of solid models is required.

Robot designer is a computer with peripheral devices, tools, including interface, description languages, database management systems, CAD systems and a knowledge base as a set of thesauri and databases of rules and procedures corresponding to certain design scenarios.

Figure 2 shows a block diagram of the robot designers operation [4]. It is assumed that the robot designer can work in automatic mode or as an assistant to a designer-human, and the degree of human intervention in the process of calculation is not constant and depends on qualification of a particular user. In other words, each operator gets an operation scenario of interaction, generated beforehand or dynamically. Operation scenario comprises the degree of automation, the preferred choice of input-output data, the need to perform various stages of the calculation and so on.

Thus, the interface of the robot-designer depends on the preferences of the particular user.

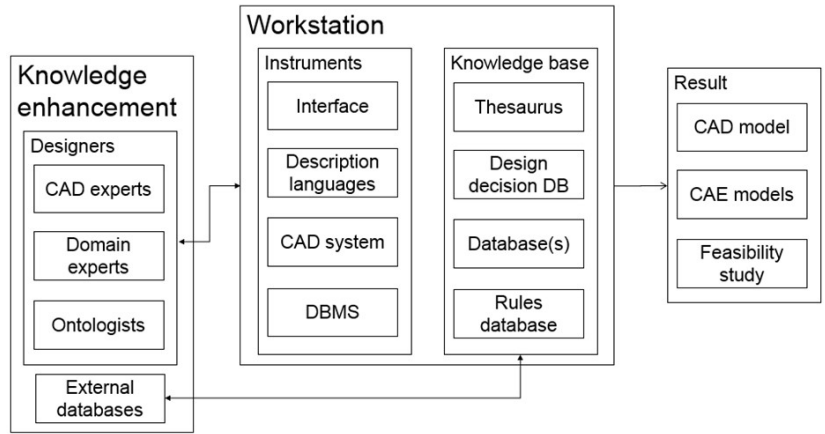

Fig. 2. Robot-designer's operation scheme

\section{INTERFACE OF THE SYSTEM}

The interface, described in the paper, is intended for an aircraft preliminary design system based on a meta-model of the aircraft (created in the CATIA CAD system) and a database (currently based on MS Excel). The overview of the systems interface is presented on figure 3 .

The interface consists of several major parts: project timeline (1), interactive section (2), general information window (3) and the model preview window (4).

The timeline represents the scenario of creating an aircraft on a high level of abstraction. Therefore, subsections of the timeline represent business processes of a lower level of abstraction. For example, for the wing design module the processes are:

1. defining the planform of the wing;

2. defining the aspect ratio of the wing;

3. defining the tapering of the wing;

4. defining the aerodynamic profile of the wing;

5. defining the parameters of roll control;

6. defining the mechanization of the wing.

Each of the processes has its own corresponding module that loads automatically based on a design scenario preset. The design scenario defines the level of the user's involvement into the designing process. Implication of the design scenarios allows for tuning of the interface towards individual needs of users within a single design environment utilizing the same interface rules and allowing, if needed, natural translation between the design scenarios [5].

\section{PARAMETERIZED CAD MODELS}

The problem of analytical design of the aircraft requires a construction of a mathematical model of the product, which is characterized by a set of external parameters (requirements of the technical project of the aircraft: the mass and other parameters of the payload, the operational range; cruising speed; class of the airfield and, accordingly, the length and width of the runway, its characteristics, category and parameters); and a set 


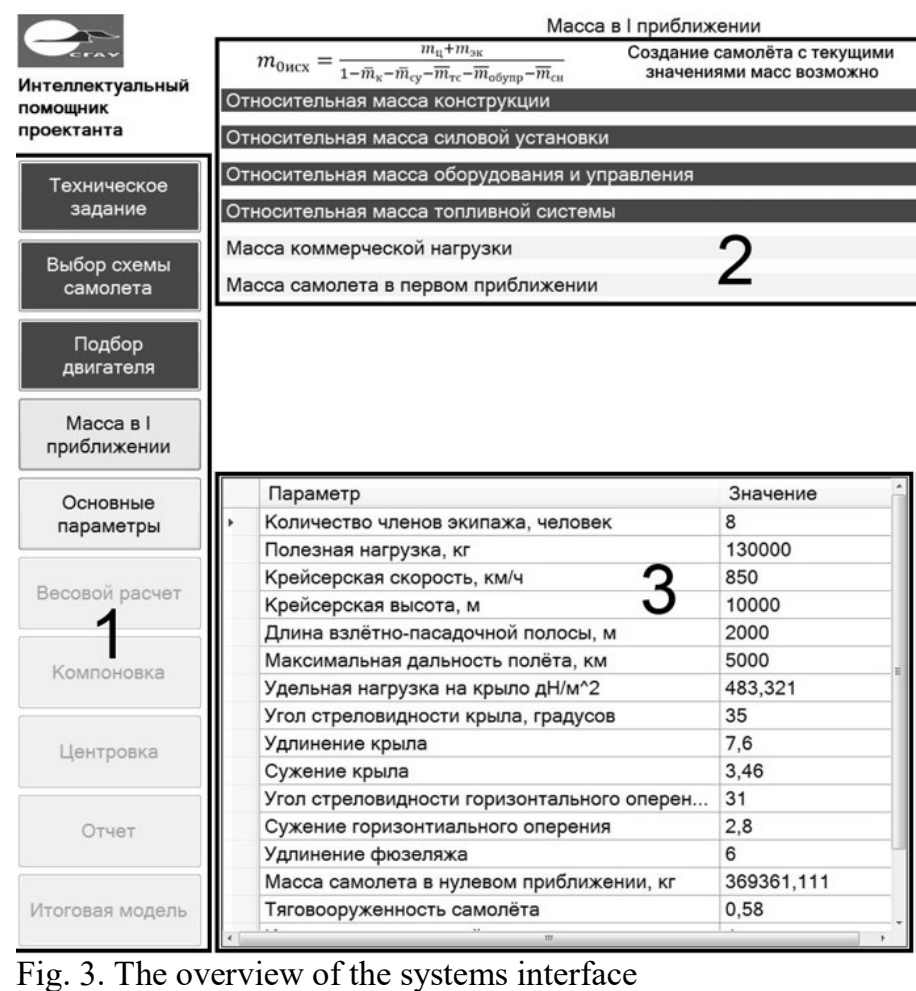

of internal parameters (parameters of the airplane defined in the process of solving the design task: first of all take-off weight, wing load, starting thrust-weight ratio, aerodynamic characteristics, the size of the aircraft and its components, structure and system parameters, and so on.), as well as objective function (criterion or performance criteria) that lets you choose among alternative projects better, providing extreme value the target function [5].

Robot designer is not able to synthesize radically different versions of structural layouts due to a number of software and hardware limitations, so the system uses the design decisions that have been previously implemented into it.

Robot designer is capable of analyzing a range of options for schemes and layouts of a passenger aircraft and, automatically, or, if required, on the basis of a dialogue with the designer, select the option that best meets the technical requirements of the customer. The robot has a modular design - individual modules, the implementation of which is initiated by the robot-designer in accordance with a given logic, solve individual subtasks (i.e., the estimation of the aspect ratio of the wing or its weight). Based on the data obtained during the designing of parts, the robotdesigner builds a parameterized three-dimensional model of the aircraft in the CATIA CAD system as shown on figure 4 .
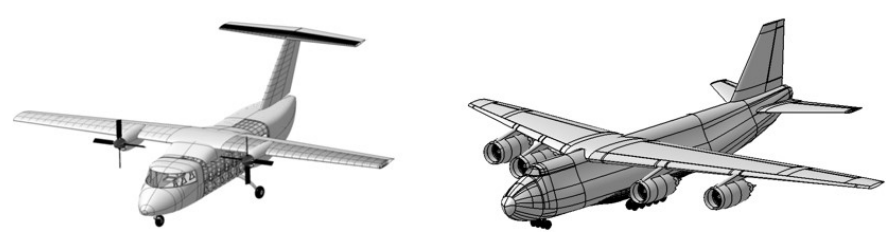

Fig. 4. CAD models of different airplanes automatically generated by the robot-designer

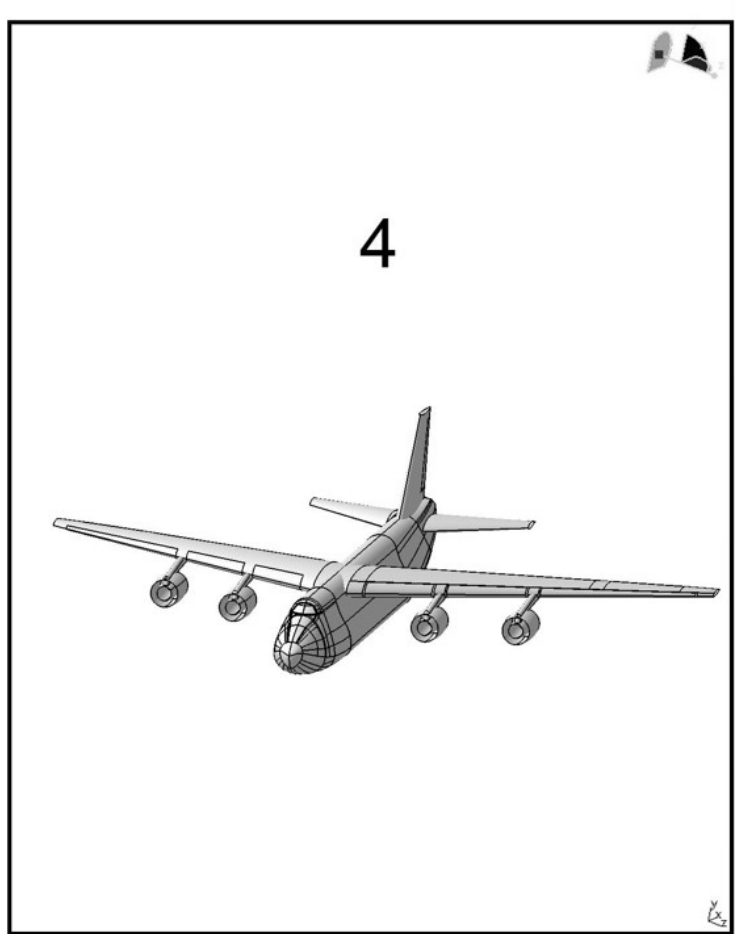

In the present study, CATIA CAD User-Defined Feature (UDF), which are a special kind of primitives CAD CATIA, were utilized as a tool for automating the creation of geometry. UDF can contain any number of elements associated with any number of CATIA automation means. For example, several data sets with formulas, rules and reactions to events could be presented as one UDF element.

The model consists of macro primitives. The airplane is separated on several macro primitives - wing, fuselage, and empennage. Each macro primitive consists of several parts, for example, fuselage consists of three components - the nose part, the tail part and the cylindrical part, each part having its own dimensional and structural characteristics depending on the design scenario. The described method can be applied to a limited number of airframe parts such as wing, fuselage and empennage, because design features of landing gear and power plant are determined by the commercial requirements, operation conditions and other specific factors beyond the scope of aircraft structure analysis.

Certain projects within the robot-designer are carried out for several years. With each consecutive year students add details to the model, describing more features and getting more feasible results. The evolution of a model of a heavy transport airplane is shown on figure 5 .

The aerodynamic calculations start after obtaining the first CAD models of geometry. Generated models are loaded into a desired CAE of CFD system (i.e. ANSYS) for the analysis of aerodynamic loads, as shown on figure 6.

Calculated aerodynamic loads are later used for the structural analysis of the airframe as shown on figure 7. 


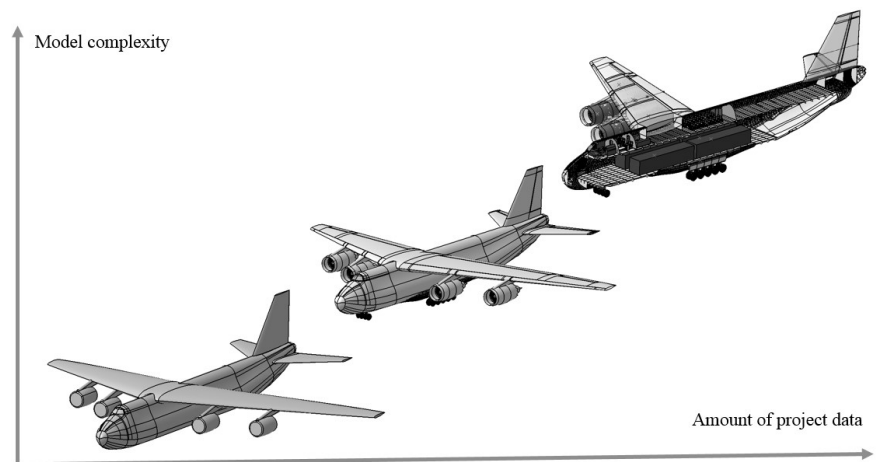

Fig. 5. Airplane model's fidelity evolution

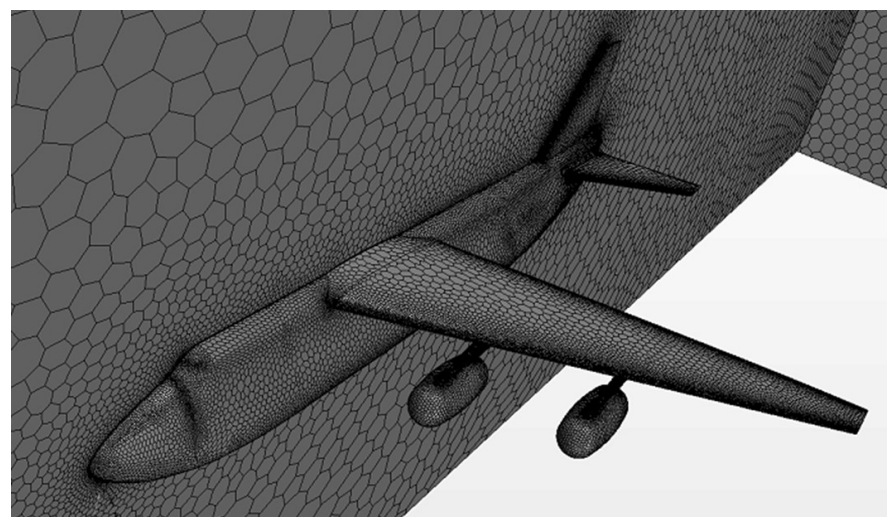

Fig. 6. Aerodynamic CAE analysis based on airplane's geometric model

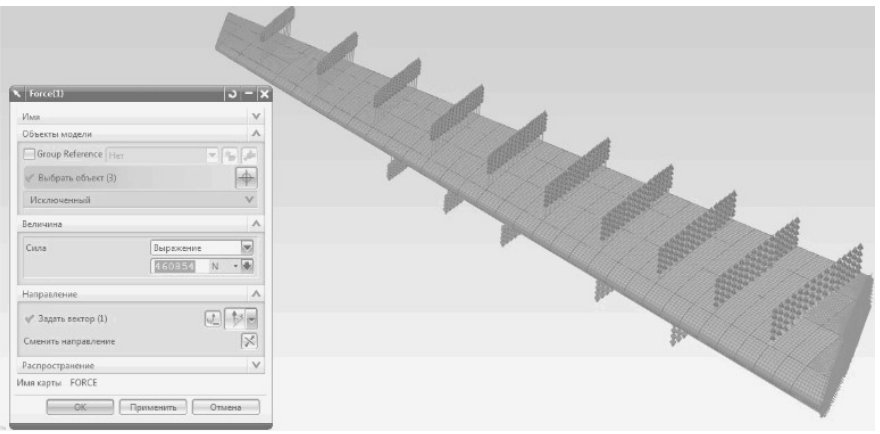

Fig. 7. Structural CAE analysis of the airplane's wing

\section{RESUlts VALIDATION}

Parameterized CAD models, developed by the masters students, need verification. In order to determine validity of the designed models, each model is tested in comparison to a number of similar airplanes, designed by specialist students as their semester project. Each of those projects corresponds to a different set of starting design parameters and the automated model must obtain result, similar to the student's. Specialist students conduct their work in a form of weekly laboratory courses, where every step of their project is being evaluated by tutors thus minimizing the possibility of rough mistakes. If the created automated model hold up to the test, it is accepted as a part of the robot-designer as a possible design solution.

\section{AERODYNAMIC EXPERIMENTS}

The results of the aerodynamic testing, obtained using CFD software are checked using the university wind tunnel. A model of the airplane is manufactured using a rapid prototyping system (a 3D printer) and is placed into the wind tunnel on the aerodynamic scale, as shown on figure 8 . Measurements taken during the experiment serve to validate the results of the $\mathrm{CAE}$ and CFD modelling.

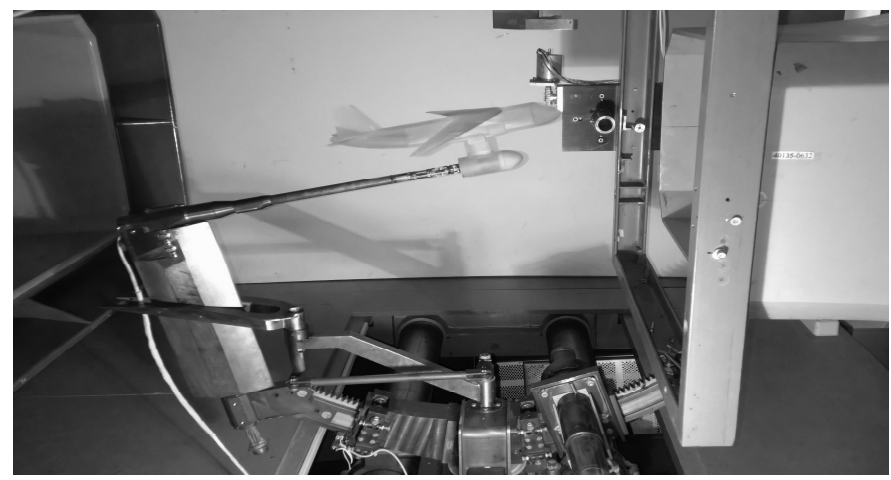

Fig. 8. Aerodynamic tests performed on a scale model obtained via rapid prototyping hardware

\section{VIII.CONCLUSION}

The results obtained with a prototype robot designer in the preliminary design of the aircraft, demonstrating the feasibility of design automation systems based on parameterized CAD models in the design of complex technical artefacts. The presented prototype allows users to successfully create threedimensional model of the aircraft and its components, as well as their various representations, automatically performing complex actions. Thesaurus is used to attribute models parameters and simultaneously serves as the prime source of terms and definitions for the HLP generation interface and a means of linking of terms and parameters from databases, containing information about past projects.

\section{REFERENCES}

[1] Wriggers, P., Siplivaya, M., Joukova, I. and Slivin, R.: Intelligent support of engineering analysis using ontology and case-based reasoning, Engineering Applications of Artificial Intelligence, 20 (2008), pp. 709720.

[2] Linköping Studies in Science and Technology. Dissertations, Linköping University. No. 1479. 2012. - $116 \mathrm{p}$.

[3] Dattoma, V., De Giorgi, M., Giancane, S., Manco, P., Morabito, A.E.: A parametric-associative modeling of aeronautical concepts for structural optimization, Adv. Eng. Software. 2012;50(1):97-109

[4] Borgest, N.M, Gromov An.A., Gromov Al.A., Korovin M.D.: The concept of automation in conventional systems creation applied to the preliminary aircraft design. A. Wili'nski et al. (eds.), Soft Computing in Computer and Information Science,Advances in Intelligent Systems and Computing 342, Springer International Publishing Switzerland 2015, pp 155-165.DOI 10.1007/978-3-319-15147-2_13

[5] Liersch, C., Hepperle, M.: A Unified Approach for Multidisciplinary PreliminaryAircraft Design, CEAS European Air and Space Conference, 26-29 October 2009, Manchester, UK (2010) 